\title{
Beneficial Effects of Dilazep on the Palmitoyl-L-carnitine-Induced Derangements in Isolated, Perfused Rat Heart: Comparison with Tetrodotoxin
}

\author{
Akiyoshi Hara, Johji Arakawa, Hiroko Hashizume and Yasushi Abiko \\ Department of Pharmacology, Asahikawa Medical College, Asahikawa 078, Japan
}

Received January 27, 1997 Accepted March 10, 1997

\begin{abstract}
The present study was carried out to determine the effect of dilazep, having an inhibitory effect on the $\mathrm{Na}^{+}$channel, on the mechanical dysfunction and metabolic derangements induced by palmitoyl-L-carnitine in isolated rat heart and to compare the effect of dilazep with that of tetrodotoxin, a specific inhibitor of the $\mathrm{Na}^{+}$channel. Rat hearts were perfused aerobically at a constant flow according to Langendorff's technique and paced electrically. Palmitoyl-L-carnitine $(5 \mu \mathrm{M})$ decreased the left ventricular developed pressure and increased the left ventricular end diastolic pressure (i.e., it produced mechanical dysfunction), decreased the tissue level of adenosine triphosphate and increased the tissue level of adenosine monophosphate (i.e., it produced metabolic derangements). These mechanical and metabolic alterations induced by palmitoyl-L-carnitine were attenuated by either dilazep $(1 \mu \mathrm{M})$ or tetrodotoxin $(3 \mu \mathrm{M})$. On the other hand, neither dilazep nor tetrodotoxin modified the mechanical function and energy metabolism of the normal (palmitoyl-L-carnitine-untreated) heart. These results suggest that inhibition of the $\mathrm{Na}^{+}$channel with dilazep or tetrodotoxin is responsible, at least in part, for attenuating the palmitoyl-I-carnitineinduced mechanical dysfunction and metabolic derangements in the heart.
\end{abstract}

Keywords: Dilazep, Tetrodotoxin, Long-chain acylcarnitine, $\mathrm{Na}^{+}$channel, heart (rat)

During myocardial ischemia, the tissue levels of longchain acylcarnitines, such as palmitoyl-t-carnitine, increase markedly because of inhibition of mitochondrial $\beta$-oxidation of free fatty acids $(1,2)$. Long-chain acylcarnitines that have accumulated in the tissue are preferentially incorporated into phospholipid bilayers of the sarcolemmal membrane and cause a disturbance in the homeostasis of ions in the cardiac cells $(1,2)$. Therefore, long-chain acylcarnitines are considered to be one of the factors involved in production of irreversible damage in the ischemic heart (1-4). In fact, palmitoyl-L-carnitine produces severe mechanical dysfunction (5), electrophysiological alterations $(4,6,7)$ and membrane dysfunction (8) in the heart. This view can be supported by the fact that inhibitors of carnitine acyltransferase $\mathbf{I}$ attenuate both tissue accumulation of long-chain acylcarnitines and myocardial ischemic derangements in situ (3) and in vitro $(4,9)$.

Recently we found that dilazep, a coronary dilator producing a cardioprotective effect, attenuates mechanical dysfunction induced by exogenous palmitoyl-I-carni- tine in the isolated rat heart (10). The action of dilazep to attenuate the palmitoyl-L-carnitine-induced derangements may contribute to its cardioprotective action against ischemia-reperfusion damage. One of the characteristic pharmacological properties of dilazep is reported to be potentiation of the cardiovascular effects of adenosine through inhibition of adenosine uptake into cardiac and endothelial cells $(5,11,12)$. The beneficial action of dilazep on the palmitoyl-L-carnitine-induced mechanical dysfunction, however, is not due to potentiation of the cardiovascular effects of adenosine but due to unknown actions (10). Interestingly, dilazep has a potent blocking action on the $\mathrm{Na}^{+}$channel in cardiac cells $(13,14)$, and palmitoyl-t-carnitine causes opening of $\mathrm{Na}^{+}$channels in cardiomyocytes $(6,7)$. These facts lead to a view that the beneficial effect of dilazep on the palmitoyl-L-carnitineinduced mechanical dysfunction may be due to inhibition of the cardiac $\mathrm{Na}^{+}$channel, although it is unclear whether inhibition of the $\mathrm{Na}^{+}$channel is important in attenuating the palmitoyl-L-carnitine-induced mechanical dysfunction. In the present study, therefore, the effect of 
tetrodotoxin, a specific inhibitor of the $\mathrm{Na}^{+}$channel, on mechanical dysfunction induced by palmitoyl-L-carnitine was studied in the isolated rat heart, and compared with that of dilazep. We also examined the effects of dilazep and tetrodotoxin on the palmitoyl-L-carnitine-induced changes in energy metabolism in the heart because palmitoyl-L-carnitine has a harmful effect on the energy metabolism (15).

\section{MATERIALS AND METHODS}

\section{Heart perfusion}

The protocol of animal experiments in the present study was approved by the "Asahikawa Medical College Committee on Animal Research". Male Sprague-Dawley rats (9- to 10-weeks-old; Sankyo Labo Service Corporation, Sapporo) were used in the present study. They were supplied with food and water ad libitum and injected with heparin $(1,000$ units $/ \mathrm{kg}$, i.p.) $30 \mathrm{~min}$ before induction of anesthesia. Under anesthesia with sodium pentobarbital (50 mg/kg, i.p.), the thorax was opened and the hearts were rapidly removed. Retrograde perfusion was started using a cannula inserted into the aorta according to the Langendorff technique. The perfusion buffer was a Krebs-Henseleit bicarbonate (KHB) buffer containing $118 \mathrm{mM} \mathrm{NaCl}, 4.7 \mathrm{mM} \mathrm{KCl}, 1.2 \mathrm{mM} \mathrm{KH} \mathrm{PO}_{4}, 1.2 \mathrm{mM}$ $\mathrm{MgSO}_{4}, 2.5 \mathrm{mM} \mathrm{CaCl}, 25 \mathrm{mM} \mathrm{NaHCO}$ and $11 \mathrm{mM}$ glucose, equilibrated with a gas mixture of $95 \% \mathrm{O}_{2}+5 \%$ $\mathrm{CO}_{2}$ and maintained at $37^{\circ} \mathrm{C}$. The oxygen tension of the buffer measured by a blood gas analyzer (Model 813; Instrumentation Laboratory, Lexington, MA, USA) was about $550 \mathrm{mmHg}$. The hearts were initially perfused at a constant perfusion pressure of $80 \mathrm{cmH}_{2} \mathrm{O}$. About 10 min after constant pressure perfusion, perfusion was switched to a constant flow perfusion $(10 \mathrm{ml} / \mathrm{min})$ using a microtube pump (Eyela MP-3; Tokyo-Rikakikai Instruments, Tokyo), and this flow rate was maintained constant throughout the experiment. The heart rate was kept constant by pacing the heart at 300 beats $/ \mathrm{min}$ with an electronic stimulator (3F46; San-Ei Instruments, Tokyo) during the course of the study. Rectangular pulses having 2-msec duration with the voltage of $6 \mathrm{~V}$ (about 3 times the threshold voltage) were applied to the left ventricle for pacing of the heart.

As indices of mechanical function, left ventricular systolic pressure (LVSP), left ventricular end-diastolic pressure (LVEDP) and left ventricular developed pressure (LVDP) were employed. The values of LVSP and LVEDP were determined from the left ventricular pressure (LVP) curves recorded continuously during the course of the study, and the LVDP value was calculated as LVSP minus LVEDP. For measurement of LVP, a saline-filled polyethylene cannula, connected to a pressure transducer, was inserted into the left ventricular cavity via the left atrium. Before starting the experiments, the heart was allowed to stabilize for $20 \mathrm{~min}$ under the conditions of constant flow perfusion.

\section{Experimental protocol}

The effect of dilazep or tetrodotoxin on the mechanical function and energy metabolism was examined in both palmitoyl-L-carnitine-treated heart and normal heart experiments. In the normal heart experiments, the heart was not treated with palmitoyl-L-carnitine. Dilazep, tetrodotoxin or vehicle (KHB buffer for both dilazep and tetrodotoxin) was infused into the aortic cannula for 45 $\mathrm{min}$ at a constant flow rate of $0.1 \mathrm{ml} / \mathrm{min}$. In the palmitoyl-L-carnitine-treated heart experiments, palmitoyl-L-carnitine was infused into the aortic cannula at the constant flow rate of $0.1 \mathrm{ml} / \mathrm{min}$ for $10 \mathrm{~min}$ from 10 min after starting the infusion of dilazep, tetrodotoxin or vehicle. The experimental conditions and protocol of the normal heart experiments were essentially the same as in the palmitoyl-L-carnitine-treated heart experiments, except for an infusion of KHB buffer solution instead of palmitoyl-L-carnitine solution. In each group, LVSP, LVEDP and LVDP were continuously recorded before and during the infusion of dilazep, tetrodotoxin or vehicle. To measure the tissue levels of high-energy phosphates, the heart was frozen at the end of the observation period ( $45 \mathrm{~min}$ after the start of infusion of dilazep, tetrodotoxin or vehicle) with freezing clamps previously chilled in liquid nitrogen.

\section{Biochemical analysis}

The frozen myocardial samples were stored in liquid nitrogen (at $-173^{\circ} \mathrm{C}$ ) until the biochemical analysis was performed. The frozen myocardial sample was pulverized in a mortar cooled with liquid nitrogen. To measure the tissue water content and dry weight of the tissue, a part of the pulverized tissue powder was weighed and put into an oven and allowed to stand in it overnight. The remainder of the tissue powder was used for determination of the tissue levels of adenosine triphosphate (ATP), adenosine diphosphate (ADP), adenosine monophosphate (AMP) and creatine phosphate ( $\mathrm{CrP})$.

ATP, ADP, AMP and CrP were extracted from the pulverized tissue sample with $6 \%$ perchloric acid. The tissue extract was centrifuged at $10,000 \times g$ for $10 \mathrm{~min}$ at $4^{\circ} \mathrm{C}$. The supernatant was neutralized by $70 \% \mathrm{KOH}$ and centrifuged at $10,000 \times \mathrm{g}$ for $10 \mathrm{~min}$ at $4^{\circ} \mathrm{C}$ again. The resultant solution was used for determination of ATP, ADP, AMP and CrP. These metabolites were measured according to the standard enzymatic procedures (16) using a spectrophotometer (Gilford system 2600; Gilford Instrument Laboratories Inc., Oberlin, OH, USA). 


\section{Drugs}

Dilazep dihydrochloride (Kowa Pharmaceutical Co., Ltd., Tokyo), tetrodotoxin (Sankyo Co., Ltd., Tokyo) and palmitoyl-L-carnitine (Sigma Chemical Company, St. Louis, MO, USA) were dissolved in KHB buffer solution. Dilazep, tetrodotoxin or vehicle (KHB buffer solution) was infused at a flow rate of $0.1 \mathrm{ml} / \mathrm{min}$, by an infusion pump, into the inflow tube connected to the side arm of the aortic cannula. Palmitoyl-L-carnitine or KHB buffer solution was also infused to the inflow tube at a flow rate of $0.1 \mathrm{ml} / \mathrm{min}$ with another infusion pump. The final concentration of dilazep in the perfusate (KHB buffer perfused at a flow rate of $10 \mathrm{ml} / \mathrm{min}$ plus dilazep solution infused at a flow rate of $0.1 \mathrm{ml} / \mathrm{min}$ ) was set to 0.1 or 1 $\mu \mathrm{M}$. Nevertheless, the concentration of dilazep changed from 0.1 to $0.099 \mu \mathrm{M}$ or 1 to $0.99 \mu \mathrm{M}$ when palmitoyl-Lcarnitine was added to the perfusate because the total flow rate of perfusate increased from $10.1 \mathrm{ml} / \mathrm{min}$ to $10.2 \mathrm{ml} / \mathrm{min}$ during the palmitoyl-L-carnitine infusion. Tetrodotoxin was also infused into the aortic cannula at a flow rate of $0.1 \mathrm{ml} / \mathrm{min}$ so that the final concentration of tetrodotoxin in the perfusate was 1 or $3 \mu \mathrm{M}$, although the concentration of tetrodotoxin changed from 1 to $0.99 \mu \mathrm{M}$ or 3 to $2.97 \mu \mathrm{M}$ during the infusion of palmitoyl-L-carnitine. These small changes in the concentration of dilazep or tetrodotoxin were about $\mathbf{1 \%}$ and did not affect LVP. The final concentration of palmitoyl-L-carnitine in the perfusate including infusion solution was $5 \mu \mathrm{M}$. The reagents and enzymes used for biochemical analysis were purchased from Sigma Chemical Company.

\section{Statistical analyses}

All values are expressed as means \pm S.E.M. When mechanical data were compared between vehicle-treated and drug-treated groups, statistical analysis was performed with a two-way repeated measures ANOVA followed by Dunnett's test for multiple comparison. When the metabolic data were compared between vehicle and palmitoyl-L-carnitine + vehicle groups, the unpaired Student's $t$-test was employed. When metabolic data were compared between vehicle-treated and drug-treated groups, statistical analysis was performed with a one-way ANOVA followed by Dunnett's test for multiple comparison. A difference was considered statistically significant at $\mathrm{P}<0.05$.

\section{RESULTS}

\section{Mechanical function}

In the normal heart, dilazep $(0.1$ and $1 \mu \mathbf{M})$ or tetrodotoxin ( 1 and $3 \mu \mathrm{M}$ ) did not have any effect on the LVSP ( $P>0.05$ by ANOVA for each concentration of dilazep or tetrodotoxin) and LVEDP ( $P>0.05$ by

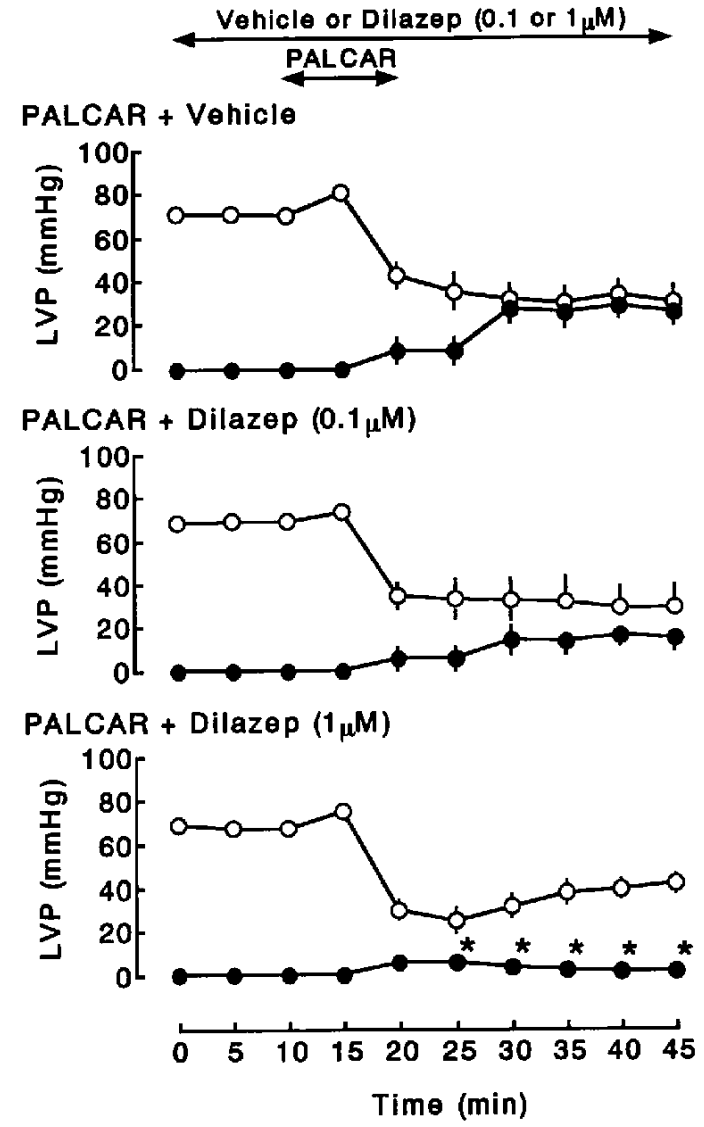

Fig. 1. Effect of dilazep on the palmitoyl-L-carnitine (PALCAR)induced changes in mechanical function. Changes of LVP were measured in the PALCAR + vehicle $(\mathrm{n}=8)$, PALCAR + dilazep $(0.1 \mu M)(n=6)$ and PALCAR + dilazep $(1 \mu M)(n=6)$ groups. Open circles indicate LVSP and solid circles indicate LVEDP. Starting time of vehicle or dilazep infusion was taken as 0 min. Vehicle or dilazep ( 0.1 or $1 \mu \mathrm{M}$ as the final concentration in perfusate) was infused from 0 to $45 \mathrm{~min}$. PALCAR $(5 \mu \mathrm{M}$ as the final concentration in perfusate) was infused for $10 \mathrm{~min}$ from $10 \mathrm{~min}$ after the start of vehicle or dilazep infusion. Each value represents a mean \pm S.E.M. ${ }^{*} \mathrm{P}<0.05$, when compared with the value in the PALCAR + vehicle group.

ANOVA for each concentration of dilazep or tetrodotoxin) during the entire course of the study (data not shown). Figures 1 and 2 show the effects of dilazep and tetrodotoxin, respectively, on the palmitoyl-L-carnitineinduced changes in LVSP and LVEDP. In the palmitoylL-carnitine-treated heart, there was no significant difference in the values of LVSP and LVEDP before starting the palmitoyl-L-carnitine infusion $(0,5$ and $10 \mathrm{~min}$ in Figs. 1 and 2 ) between the palmitoyl-L-carnitine + vehicle and palmitoyl-L-carnitine + dilazep groups and between the palmitoyl-L-carnitine + vehicle and palmitoyl-L-carnitine + tetrodotoxin groups. In the palmitoyl-t-carnitine + vehicle group, palmitoyl-L-carnitine produced a longlasting decrease in LVSP $(\mathbf{P}<0.001$ by ANOVA) follow- 


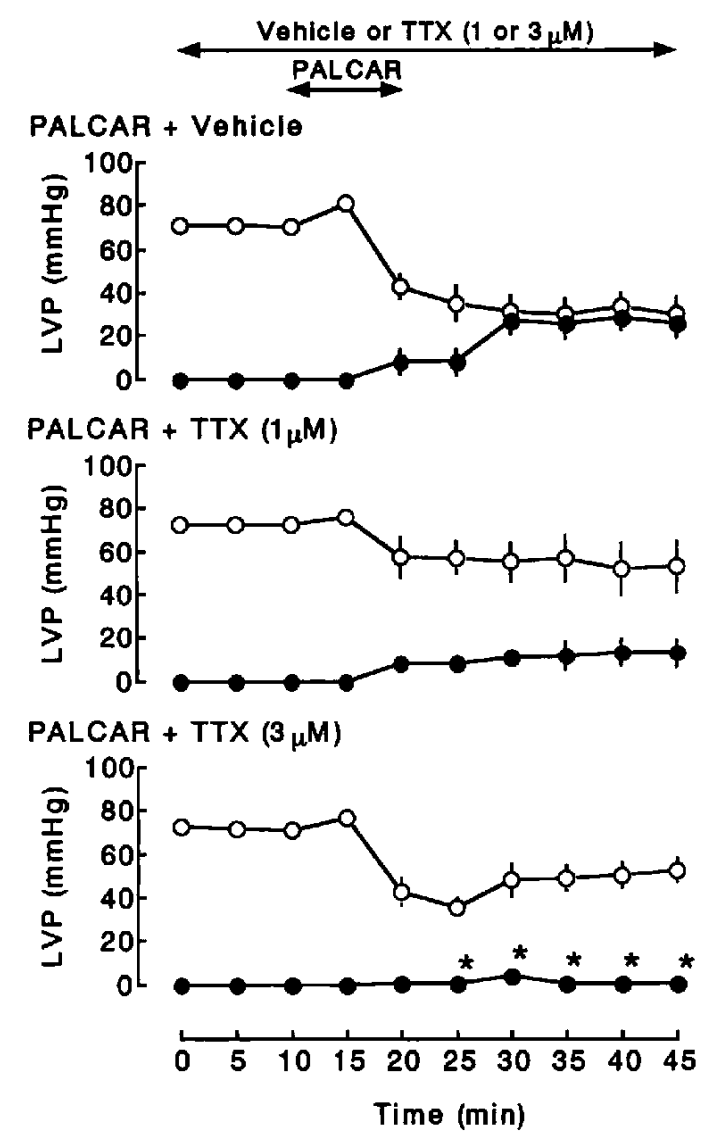

Fig. 2. Effect of tetrodotoxin (TTX) on the palmitoyl-L-carnitine (PALCAR)-induced changes in mechanical function. Changes of LVP were measured in the PALCAR + vehicle $(n=8)$, PALCAR + TTX $(1 \mu \mathrm{M})(n=6)$ and PALCAR + TTX $(3 \mu \mathrm{M})(\mathrm{n}=5)$ groups. Open circles indicate LVSP and solid circles indicate LVEDP. Starting time of vehicle or TTX infusion was taken as $0 \mathrm{~min}$. Vehicle or TTX ( 1 or $3 \mu \mathrm{M}$ as the final concentration in perfusate) was infused from 0 to $45 \mathrm{~min}$. PALCAR ( $5 \mu \mathrm{M}$ as the final concentration in perfusate) was infused for $10 \mathrm{~min}$ from $10 \mathrm{~min}$ after start of vehicle or TTX infusion. Data on the PALCAR + vehicle group are the same as those in Fig. 1. Each value represents a mean \pm S.E.M. ${ }^{*} \mathrm{P}<0.05$, when compared with the value in the PALCAR + vehicle group.

ing a slight and transient increase in LVSP and a longlasting increase in LVEDP $(P<0.001$ by ANOVA). Neither dilazep nor tetrodotoxin modified the decrease in LVSP induced by palmitoyl-L-carnitine throughout the experiment ( $P>0.05$ compared with the palmitoyl-L-carnitine + vehicle group). Low concentrations of dilazep $(0.1 \mu \mathrm{M})$ and tetrodotoxin $(1 \mu \mathrm{M})$ failed to attenuate the increase in LVEDP induced by palmitoyl-L-carnitine. However, the increase in LVEDP induced by palmitoylL-carnitine was attenuated almost completely by dilazep $(1 \mu \mathrm{M})$ or tetrodotoxin $(3 \mu \mathrm{M})$ at 25 to 45 min after starting the drug infusion $(P<0.05$ compared with the palmitoyl-L-carnitine + vehicle group).
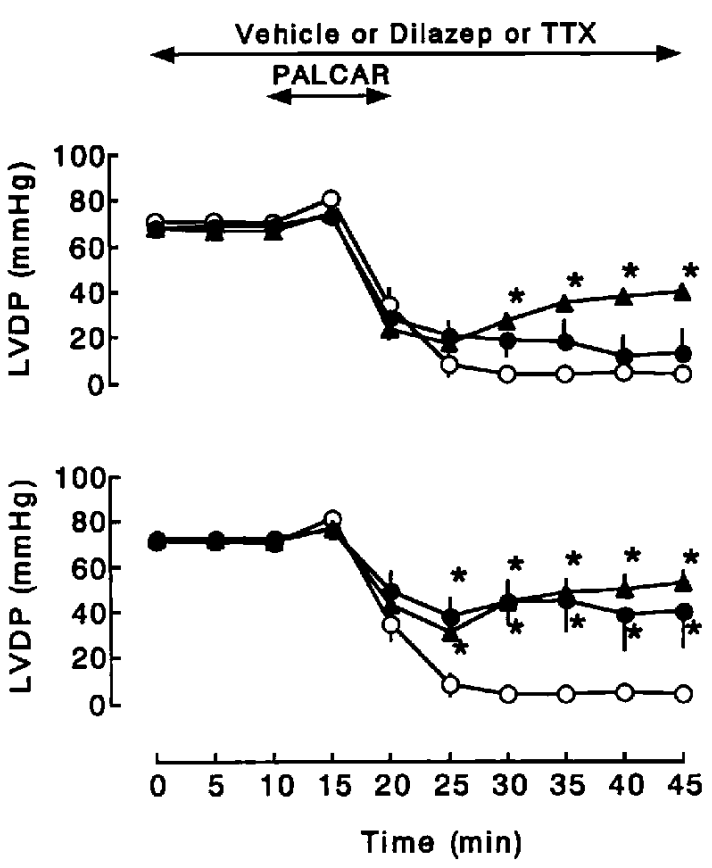

Fig. 3. Effect of dilazep (upper panel) or tetrodotoxin (TTX) (lower panel) on the palmitoyl-L-carnitine (PALCAR)-induced changes in LVDP. Upper panel shows the changes of LVDP in the PALCAR + vehicle $(\bigcirc)$, PALCAR + dilazep $(0.1 \mu \mathrm{M})(O)$ and PALCAR + dilazep $(1 \mu \mathrm{M})$ (A) groups. Lower panel shows the changes of LVDP in the PALCAR + vehicle (O), PALCAR + TTX $(1 \mu \mathrm{M})$ (O) and PALCAR + TTX (3 $\mu \mathrm{M})(\mathbf{A})$ groups. Values were calculated from the values of LVSP and LVEDP in Figs. 1 and 2. Control data (PALCAR + vehicle) in upper and lower panels are obtained from the same hearts. Each value represents a mean \pm S.E.M. ${ }^{*} \mathrm{P}<0.05$, when compared with the value in the PALCAR + vehicle group.

Figure 3 shows the effect of dilazep or tetrodotoxin on the palmitoyl-L-carnitine-induced changes in LVDP. Before starting the palmitoyl-L-carnitine infusion $(0,5$ and $10 \mathrm{~min}$ in Fig. 3), there was no significant difference in the LVDP value among all the groups. In the palmitoyl-L-carnitine + vehicle group, palmitoyl-L-carnitine decreased LVDP markedly ( $P<0.001$ by ANOVA) because of the decrease in LVSP and the increase in LVEDP. Dilazep $(1 \mu \mathrm{M})$ significantly attenuated the palmitoyl-L-carnitine-induced decrease in LVDP at 30 to 45 min after starting the dilazep infusion $(P<0.05$ compared with the palmitoyl-L-carnitine + vehicle group), although a low concentration of dilazep $(0.1 \mu \mathrm{M})$ did not. Tetrodotoxin ( 1 and $3 \mu \mathrm{M}$ ) also attenuated the palmitoyl$\mathrm{L}$-carnitine-induced decrease in LVDP at 25 to $45 \mathrm{~min}$ after starting the tetrodotoxin infusion $(P<0.05$, compared with the palmitoyl-L-carnitine + vehicle group). These results indicate that dilazep and tetrodotoxin attenuate the mechanical dysfunction induced by palmitoyl-L-carnitine. 
Normal (PALCAR-untreated) heart
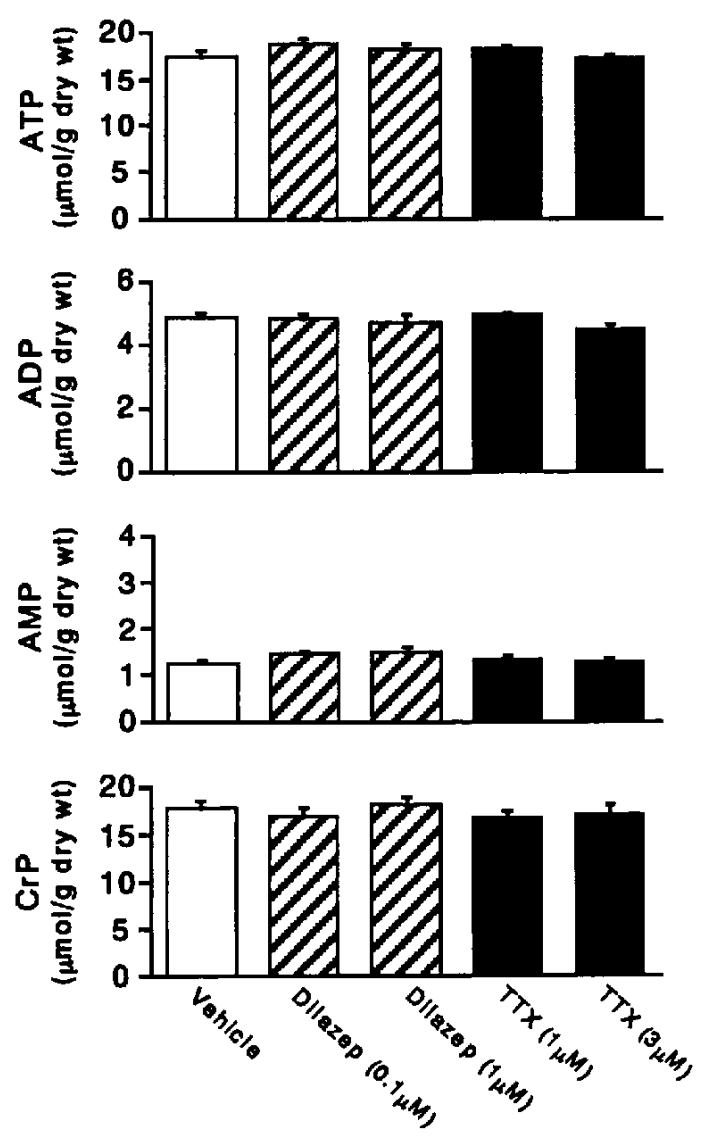

PALCAR-treated heart
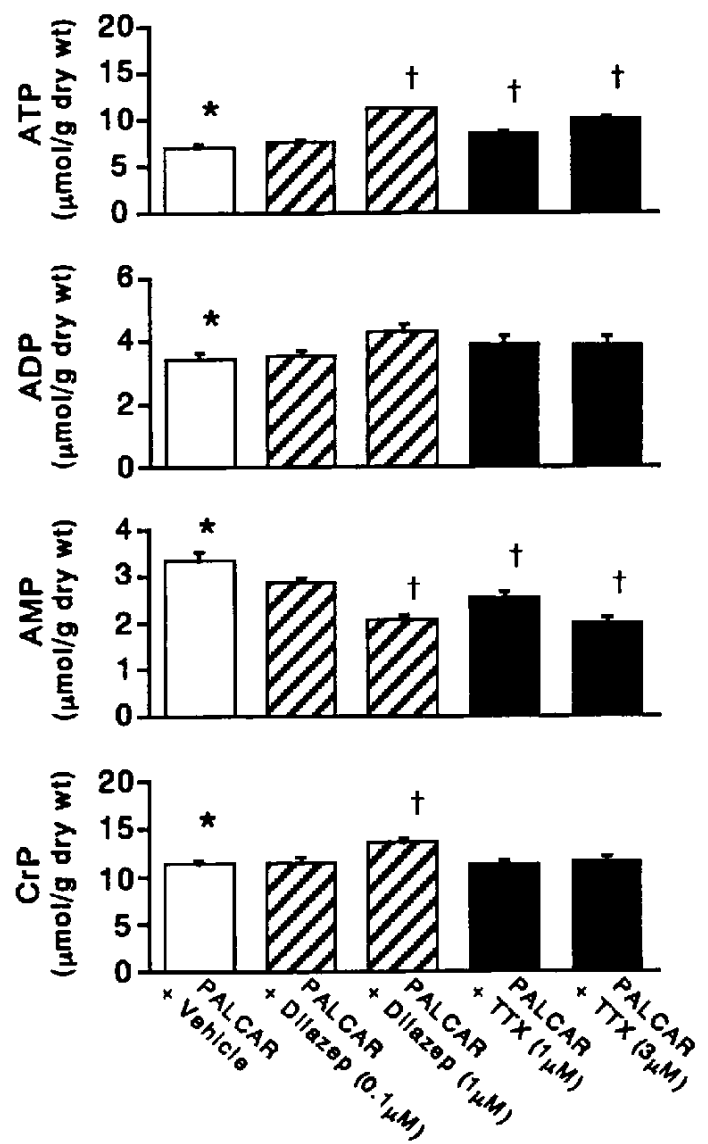

Fig. 4. Effect of dilazep or tetrodotoxin (TTX) on the palmitoyl-L-carnitine (PALCAR)-induced changes in the tissue levels of high-energy phosphates. The tissue levels of ATP, ADP, AMP and $\mathrm{CrP}$ at the end of experiments (45 min after start of dilazep, TTX or vehicle infusion) were measured in the normal (PALCAR-untreated) heart (left four panels) and PALCAR-treated heart (right four panels). Hearts are those in Figs. 1 and 2 . Each value represents a mean \pm S.E.M. ${ }^{*} P<0.05$, when compared with the value in the vehicle group. ${ }^{\dagger} \mathrm{P}<0.05$, when compared with the value in the PALCAR + vehicle group.

\section{Energy metabolism}

Figure 4 shows the tissue levels of ATP, ADP, AMP and $\mathrm{CrP}$ at the end of experiments in the normal and palmitoyl-I-carnitine-treated hearts. In the normal heart, the tissue levels of ATP, ADP, AMP and CrP were similar between the vehicle and each of the drug-treated groups. In the palmitoyl-L-carnitine + vehicle group, palmitoyl-L-carnitine decreased the tissue levels of ATP, ADP and CrP and increased the tissue level of AMP $(\mathrm{P}<0.05$ between the vehicle and palmitoyl-L-carnitine + vehicle groups). The palmitoyl-L-carnitine-induced changes in the tissue levels of ATP and AMP, however, were attenuated significantly by dilazep $(1 \mu \mathrm{M})$ or tetrodotoxin ( 1 and $3 \mu \mathrm{M})(\mathrm{P}<0.05$ compared with the palmitoyl-L-carnitine + vehicle group). Dilazep $(1 \mu \mathrm{M})$ also slightly but significantly attenuated the decrease in tissue level of $\mathrm{CrP}$ induced by palmitoyl-L-carnitine $(\mathrm{P}<0.05$, compared with the palmitoyl-I-carnitine + ve- hicle group). Nevertheless, a low concentration of dilazep $(0.1 \mu \mathrm{M})$ failed to attenuate the palmitoyl-L-carnitineinduced changes in the tissue levels of high-energy phosphates. These results indicate that both dilazep and tetrodotoxin attenuate the metabolic derangements induced by palmitoyl-L-carnitine.

\section{DISCUSSION}

In the present study, the effect of dilazep or tetrodotoxin on the palmitoyl-L-carnitine-induced mechanical and metabolic derangements was examined in the isolated perfused rat heart. The concentration of palmitoyl-Lcarnitine was chosen as $5 \mu \mathrm{M}$ for the following three reasons: First, this is the submaximal concentration that produces a long-lasting mechanical dysfunction (10). Second, administration of $5 \mu \mathrm{M}$ palmitoyl-L-carnitine may inflict damage to the heart to an extent similar to 
that induced by ischemia in terms of tissue accumulation of long-chain acylcarnitines (8). Last, $5 \mu \mathrm{M}$ palmitoyl-Lcarnitine produces not only mechanical dysfunction and energy deficiency but also membrane damage (8), and it induces electrophysiological alterations $(1,3)$ in the heart. We observed that palmitoyl-L-carnitine produced mechanical dysfunction (as evidenced by an increase in LVEDP and a decrease in LVDP) and metabolic damage (as evidenced by a decrease in the tissue ATP level and an increase in the tissue AMP level). These alterations induced by palmitoyl-L-carnitine were attenuated by dilazep $(1 \mu \mathrm{M})$, suggesting that dilazep has a beneficial effect on the palmitoyl-L-carnitine-induced mechanical dysfunction and metabolic derangements.

Dilazep has coronary vasodilating and negative inotropic actions through inhibition of the $\mathrm{Ca}^{2+}$ channel $(17,18)$. We have previously shown that dilazep decreases cardiac mechanical function (LVSP) at concentrations of $2 \mu \mathrm{M}$ or higher (19). In the present study, however, we used concentrations of dilazep $(0.1$ and $1 \mu \mathrm{M})$ that did not modify the mechanical function of the normal heart. In addition, dilazep would not change coronary flow, because the hearts were perfused at a constant flow rate. It is unlikely, therefore, that the protective effect of dilazep against the palmitoyl-L-carnitine-induced myocardial derangements is due to an increase in coronary flow and/or a decrease in energy consumption of the heart. In fact, dilazep did not have any effect on the tissue levels of high-energy phosphates in the normal heart. These results suggest that preservation of energy is not a cause of the beneficial effect of dilazep on the palmitoyl-L-carnitineinduced myocardial derangements but a result.

Dilazep has a potent inhibitory effect on the $\mathrm{Na}^{+}$channel in the cardiac cells $(13,14)$. According to Hashizume et al. (14), dilazep inhibits veratridine (a $\mathrm{Na}^{+}$channel opener)-induced hypercontracture in the rat myocytes at concentrations of $1 \mu \mathrm{M}$ or higher $\left(\mathrm{IC}_{50}=2.2 \mu \mathrm{M}\right)$. On the other hand, electrophysiological studies using whole cell voltage-clamp technique have demonstrated that palmitoyl-L-carnitine activates the slow-inactivating $\mathrm{Na}^{+}$ current to increase $\mathrm{Na}^{+}$influx and hence intracellular $\mathrm{Na}^{+}$concentration $(1,3)$. The increase in intracellular $\mathrm{Na}^{+}$concentration may accelerate the $\mathrm{Na}^{+}-\mathrm{Ca}^{2+}$ exchange system, causing intracellular $\mathrm{Ca}^{2+}$ overload, which is responsible for myocardial derangements (20). In fact, palmitoyl-L-carnitine induces intracellular $\mathrm{Ca}^{2+}$ accumulation in the cardiac myocyte (21). There is a possibility, therefore, that the beneficial effect of dilazep $(1 \mu \mathrm{M})$ on the palmitoyl-L-carnitine-induced myocardial derangements is due to inhibition of $\mathrm{Na}^{+}$influx in the cardiac cell. To examine this possibility, we studied whether tetrodotoxin, a specific inhibitor of the $\mathrm{Na}^{+}$ channel, attenuates the palmitoyl-L-carnitine-induced derangements. According to binding studies (22, 23), the concentrations of tetrodotoxin ( 1 and $3 \mu \mathrm{M}$ ) used in the present study are capable of inhibiting the $\mathrm{Na}^{+}$channel in the rat heart $\left(\mathrm{K}_{\mathrm{d}}\right.$ value $=0.3$ to $\left.1 \mu \mathrm{M}\right)$. The results indicated that tetrodotoxin $(1$ and $3 \mu \mathrm{M})$ attenuated the palmitoyl-L-carnitine-induced mechanical dysfunction and metabolic derangements. Because tetrodotoxin, as well as dilazep, did not modify the energy metabolism in the normal heart, the beneficial action of dilazep against the palmitoyl-L-carnitine-induced derangements is not due to energy-sparing action but due to other actions including inhibition of the $\mathrm{Na}^{+}$channel. The low concentration of dilazep $(0.1 \mu \mathrm{M})$ failed to attenuate the palmitoyl-L -carnitine-induced derangements, probably because of less potent inhibitory action on the $\mathrm{Na}^{+}$channel (13).

Lysophosphatidylcholine, an amphiphilic metabolite structurally similar to palmitoyl-L-carnitine, also accumulates in the ischemic myocardium $(1,2)$. The cardiac action of lysophosphatidylcholine is similar to or identical with that of palmitoyl-L-carnitine; it produces intracellular $\mathrm{Ca}^{2+}$ accumulation through long-lasting bursts of $\mathrm{Na}^{+}$channel openings (24). Recently, Hoque et al. (25) have demonstrated that dilazep attenuates the mechanical dysfunction and metabolic derangements induced by lysophosphatidylcholine in the isolated working rat heart. Kang and Leaf (26) have shown that tetrodotoxin attenuates the lysophosphatidylcholine-induced arrhythmias in rat myocytes. These findings support our results in terms of the cardioprotective effect of $\mathrm{Na}^{+}$channel inhibitors.

Hoque et al. (27) reported that dilazep ( $1 \mu \mathrm{M})$ attenuates myocardial derangements induced by ischemia and reperfusion in the isolated, working rat heart. The antiischemic action of dilazep has been considered to be due to potentiation of the cardiovascular effects of adenosine $(11,12)$. According to Meghji et al. (28), however, concentrations of dilazep required to inhibit adenosine uptake into the cardiac cell are very high (over $100 \mu \mathrm{M}$ ) in the rat when compared to other species. Nevertheless, dilazep is capable of attenuating myocardial changes induced by palmitoyl-L-carnitine at a concentration of 1 $\mu \mathrm{M}$. Therefore, we speculate that the action of dilazep to reduce the palmitoyl-L-carnitine-induced derangements contributes to its protective effect on the myocardium against ischemia-reperfusion damage.

In conclusion, dilazep attenuates both mechanical dysfunction and metabolic derangements of the heart induced by palmitoyl-L-carnitine, and the beneficial action of dilazep is not due to preservation of energy but probably due to inhibition of $\mathrm{Na}^{+}$channels of the cardiac membranes. 


\section{Acknowledgments}

The authors thank Mr. Tadahiko Yokoyama for his technical assistance and Ms. Miwa Tajima for her secretarial assistance. Dilazep was kindly supplied by Kowa Company (Tokyo).

\section{REFERENCES}

1 Corr PB, Saffitz JE and Sobel BE: Lysophospholipids, long chain acylcarnitines and membrane dysfunction in the ischaemic heart. Basic Res Cardiol 82, Supp 1, 199-208 (1987)

2 DaTorre SD, Creer MH, Pogwizd SM and Corr PB: Amphipathic lipid metabolites and their relation to arrhythmogenesis in the ischemic heart. J Mol Cell Cardiol 23, Supp I, 11-22 (1991)

3 Corr PB, Creer MH, Yamada KA, Saffitz JE and Sobel BE: Prophylaxis of early ventricular fibrillation by inhibition of acylcarnitine accumulation. J Clin Invest 83, 927-936 (1989)

4 Yamada KA, McHowat J, Yan G-X, Donahue K, Peirick J, Kléber AG and Corr PB: Cellular uncoupling induced by accumulation of long-chain acylcarnitine during ischemia. Circ Res 74, 83-95 (1994)

5 Hara A, Akahira $M$ and Abiko $Y$ : Effect of $K-7259$, a novel derivative of dilazep, on cardiovascular actions of adenosine: Comparison with dilazep. Arch Int Pharmacodyn Ther 330, 66-75 (1995)

$6 \mathrm{Wu}$ J and Corr PB: Palmitoyl carnitine modifies sodium currents and induces transient inward current in ventricular myocytes. Am J Physiol 266, H1034-H1046 (1994)

$7 \mathrm{Wu} \mathrm{J}$ and Corr PB: Palmitoylcarnitine increases $\left[\mathrm{Na}^{-}\right]_{\mathrm{i}}$ and initiates transient inward current in adult ventricular myocytes. Am J Physiol 268, H2405-H2417 (1995)

8 Busselen P, Sercu D and Verdonck F: Exogenous palmitoyl carnitine and membrane damage in rat hearts. $J$ Mol Cell Cardiol 20, 905-916 (1988)

9 Shikama H, Noshiro O, Ohta A and Ohata I: Effects of acylcarnitine-transferase inhibitors on adenine nucleotide metabolism and ischemic tissue injury in isolated perfused rat heart. Jpn Heart J 29, 723-734 (1988)

10 Hara A, Hashizume $\mathrm{H}$ and Abiko $\mathrm{Y}$ : Dilazep and its derivative, $\mathrm{K}-7259$, attenuate mechanical derangement induced by palmitoyl-L-carnitine in the isolated, perfused rat heart. J Pharmacol Exp Ther 279, 32-38 (1996)

11 Mustafa SJ: Effects of coronary vasodilator drugs on the uptake and release of adenosine in cardiac cells. Biochem Pharmacol 28, 2617-2624 (1979)

12 Van Belle H: Nucleoside transport inhibition: a therapeutic approach to cardioprotection via adenosine ? Cardiovasc Res 27, 68-76 (1993)

13 Chiba K, Hashizume H, Inagaki S-I and Abiko Y: Dilazep inhibits binding of batrachotoxin to sodium channels in canine sarcolemmal vesicles. Arch Int Pharmacodyn Ther 330, 138- 150 (1995)

14 Hashizume $H$, Akiyama $\mathrm{K}$ and Abiko $\mathrm{Y}$ : Effects of antiischemic drugs on veratridine-induced hypercontracture in rat cardiac myocytes. Eur J Pharmacol 271, 1-8 (1994)

15 Xiao C-Y, Chen M, Hara A, Hashizume $H$ and Abiko Y: Effects of palmitoyl-L-carnitine on energy and lipid metabolism in the heart (Abstract). J Mol Cell Cardiol 28, A336 (1996)

16 Bergmeyer HU: Methods of Enzymatic Analysis. pp 1777-1781, 2101-2110, 2127-2131, Academic Press, New York (1974)

17 Nakagawa $Y$, Gudenzi $M$ and Mustafa SJ: Calcium entry blocking activity of dilazep in dog coronary artery. Pharmacology 33, 148-156 (1986)

18 Nakagawa $Y$, Gudenzi $M$ and Mustafa SJ: Calcium entry blocking activity of dilazep and other adenosine potentiating compounds in guinea pig atria. Eur J Pharmacol 122, 51-58 (1986)

19 Hara A and Abiko Y: Protective effects of dilazep and its derivative, K-7259, on mechanical and metabolic derangements induced by hydrogen peroxide in the isolated perfused rat heart. J Pharmacol Exp Ther 277, 565-571 (1996)

20 Nayler WG, Panagiotopoulos S, Elz JS and Daly MJ: Calcium-mediated damage post-ischaemic reperfusion. J Mol Cell Cardiol 20, Supp II, 41-54 (1988)

21 Clarke B, Wyatt KM, May GR and McCormack JG: On the roles of long-chain acyl carnitine accumulation and impaired glucose utilization in ischaemic contracture development and tissue damage in the guinea pig heart. J Mol Cell Cardiol 28, 171 - 181 (1996)

22 Catterall WA and Coppersmith J: Pharmacological properties of sodium channels in cultured rat heart cells. Mol Pharmacol 20, 533- 542 (1981)

23 Rogart RB: High-STX-affinity vs. low-STX-affinity $\mathrm{Na}^{+}$channel subtypes in nerve, heart, and skeletal muscle. Ann NY Acad Sci 479, 402- 430 (1987)

24 Undrovinas AI, Fleidervish IA and Makielski JC: Inward sodium current at resting potentials in single cardiac myocytes induced by the ischemic metabolite lysophosphatidylcholine. Circ Res 71, 1231-1241 (1992)

25 Hoque ANE, Hoque $N$, Hashizume $H$ and Abiko $Y$ : A study on dilazep: II. Dilazep attenuates lysophosphatidylcholine-induced mechanical and metabolic derangements in the isolated, working rat heart. Jpn J Pharmacol 67, 233-241 (1995)

26 Kang JX and Leaf A: Protective effects of free polyunsaturated fatty acids on arrhythmias induced by lysophosphatidylcholine or palmitoylcarinitine in neonatal rat cardiac myocytes. Eur $\mathbf{J}$ Pharmacol 297, 97-106 (1996)

27 Hoque ANE, Hoque $N$, Hashizume $H$ and Abiko $Y$; A study on dilazep: I. Mechanism of anti-ischemic action of dilazep is not coronary vasodilation but decreased cardiac mechanical function in the isolated, working rat heart. Jpn J Pharmacol 67, 225-232 (1995)

28 Meghji P, Middleton K, Hassall CJS, Phillips MI and Newby AC: Evidence for extracellular deamination of adenosine in the rat heart. Int J Biochem 20, 1335-1341 (1988) 